
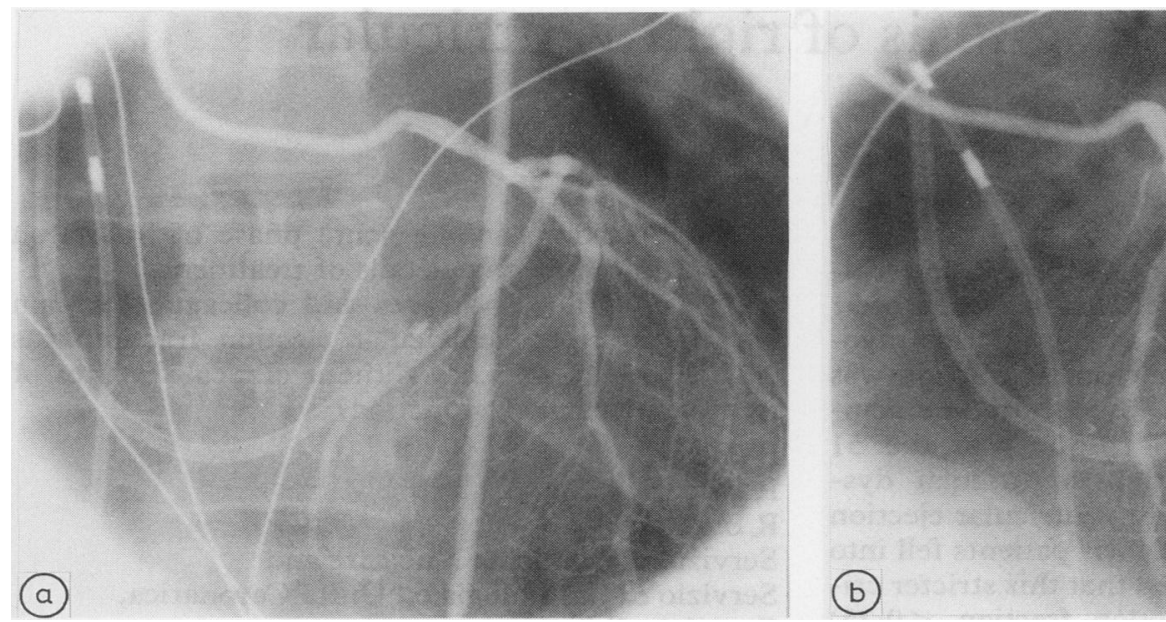

Fig 2 Cineangiograms of similar views of the left anterior descending coronary artery demonstrating successful dilatation of the stenosis. (a) Anteroposterior view; (b) left anterior oblique cranial view.

\title{
Early results after percutaneous transluminal coronary angioplasty in 400 patients
}

Sir,

Dr Sowton and coworkers $(1986 ; 56: 115-20)$ report five angioplasty related deaths in the first 400 patients who underwent percutaneous transluminal coronary angioplasty at Guy's Hospital. In 1985, one of the authors of this study, Dr Timmis, presented the preliminary results to the Junior Cardiac Club. At that time, 254 patients had undergone the procedure and there had been six deaths. The mortality figures are recorded in the minutes of the Club, which are not published or distributed but are held by the President of the Club and available for inspection by members.

I should be grateful if the authors would explain whether changes in protocol or inclusion criteria account for these discrepancies in mortality figures.

Peter Wilmshurst, Department of Cardiology, St Thomas's Hospital, London SE1 7EH.
This letter was shown to the authors, who reply as follows:

Sir,

The results in our paper were for the first angioplasty, as stated in our introduction. The presentation to the Junior Cardiac Club by $\mathrm{Dr}$ Timmis included results and complications in second or third angioplasties of patients who had recurrent angina. As also stated in our paper the results in patients who have relapse over a five year period will be presented in a separate communication.

Adam D Timmis,

Edgar Sowton,

Department of Cardiology,

Guy's Hospital,

St Thomas Street,

London SE1 9RT. 